\title{
Which Shape Characteristics of the Intermolecular Interaction of Liquid Water Determine Its Compressibility?
}

\author{
Makoto Yasutomi * \\ Department of Physics and Earth Sciences, Faculty of Science, University of the Ryukyus, Okinawa, Japan
}

We consider a fluid of spherical particles with a pair potential given by a hard core repulsion and a tail, and show that the isothermal compressibility of liquid water is determined by the degree of steepness of the soft repulsion near the hard-core contact. This helps us understand the thermodynamic mechanism that causes the compressibility anomaly of liquid water.

Keywords: isothermal compressibility, liquid water, intermolecular interaction, thermodynamic mechanism, Ornstein-Zernike approximation

\section{INTRODUCTION}

\section{OPEN ACCESS}

Edited by:

Gang Zhang

Institute of High Performance Computing, A*STAR, Singapore

Reviewed by:

Mikhail Yevgenievich Zhuravlev, Kurnakov Institute of General and Inorganic Chemistry of Russian Academy of Sciences, Russia

Junfeng Gao,

Institute of High Performance Computing, A*STAR, Singapore

*Correspondence: Makoto Yasutom g800002@lab.u-ryukyu.ac.jp

Specialty section: This article was submitted to Condensed Matter Physics, a section of the journal Frontiers in Physics

Received: 07 November 2015 Accepted: 26 April 2016 Published: 18 May 2016

Citation:

Yasutomi M (2016) Which Shape Characteristics of the Intermolecular Interaction of Liquid Water Determine Its Compressibility? Front. Phys. 4:21.

doi: 10.3389/fphy.2016.00021
It is well-known that the properties of liquid water at low temperatures change in much different ways than those exhibited by most other liquids under similar conditions [1]. These properties are determined by the interactions between water molecules, which can be derived by using thermodynamics and statistical mechanics. Therefore, it is important to study the relationship between the shape of the intermolecular interaction and each anomalous property of liquid water.

The development of realistic water models has recently shown great progress. Many of the experimentally observed anomalies of water have been reproduced in molecular dynamics or Monte Carlo simulations that use empirical force fields, albeit with significant differences in the predictions given by different models [2-11]. Among these, TIP4P/2005 probably gives the best agreement with experiment for a wide range of states and water properties [12-19]. However, no realistic models put forward up to now explains what characteristics of the intermolecular interactions determine the properties of liquid water. Realistic models can provide a description of the density and compressibility anomalies and reproduce a number of the other anomalies of water. However, the direct causes remain obscure and difficult to elucidate because such models include a number of properties of water, not all of which are related to the immediate causes. Therefore, it is impossible to capture the essential physics via the study of realistic models that include the miscellaneous properties of water, even if they can reproduce all of water's anomalies.

To capture the physics underlying a water anomaly, one should use simplified or core-softened models that include only the properties crucial to explaining that anomaly. It is important to address the mysteries individually, accumulate knowledge, and develop ideas. It is impossible to illuminate the direct causes of all the anomalies of water simultaneously. Toward this aim, a number of core-softened and simplified models have been put forward and used to perform numerical simulations. None of them [20-32]., however, has quantitatively reproduced any anomaly of liquid water or elucidated the thermodynamic mechanisms that cause the anomaly. The author believes that the self-consistent Ornstein-Zernike approximation (SCOZA) with hardcore repulsion plus Yukawa tails is presently the most useful. The SCOZA is known to describe the overall thermodynamics of liquids very well and provides a remarkably accurate critical point and coexistence curve. This scheme is entirely self-contained, which means that no supplementary thermodynamic or other input is necessary [33-40]. 
Recently, we have determined many functional representations of intermolecular interactions between water molecules that reproduce the experimentally measured densitytemperature relation at 1 bar with a reasonable accuracy by using the SCOZA [39]. Numerous similar descriptions of pair interactions will be increasingly discovered in the coming years, which will help us to understand why solid water has polymorphic structures and liquid water has a large number of anomalies.

We have also elucidated the thermodynamic mechanisms that cause the density anomaly of liquid water [40]. Our model exhibits an unusual negative thermal expansion when the value of the potential tail at the hard-core contact is in some specific range, otherwise, the density anomaly does not occur in the system. The range depends on the shape of the attraction. The thermodynamic properties of liquid water can be derived from the excess internal energy. The negative thermal expansion of liquid water below $4^{\circ} \mathrm{C}$ is induced by the behavior of excess internal energy, which is mainly determined by the large positive value of the soft repulsive tail near the hard-core contact.

Russo and Tanaka [19] used a two-state model to describe the behavior of liquid water over a wide region of the phase diagram. The first state is denoted as the $\mathrm{S}$ state, in which local structures have low energy, high specific volume, and low degeneracy. In contrast, structures in the second state are thermally excited, characterized by a high degree of disorder and degeneracy, and have low specific volume and high energy. These structures are labeled as the $\rho$ state. However, Russo and Tanaka [19] do not explain what causes the decomposition of water into the two states. We can explain the decomposition based on the thermodynamic mechanisms described in the above paragraph [40] as follows: Cooling of water generates the higher density $\rho$ state structures and increases their excess internal energies because of the high values of the soft-repulsive potential near the hard-core contact due to condensation. This causes the derivative $\alpha$ of the pressure with respect to temperature at constant density to be negative, resulting in negative thermal expansion, which generates the $S$ state structures. In this way, we can understand that the decomposition of water into the two states is not the cause of the anomaly but just an accompanying effect. Furthermore, no idea put forward up to now tells us anything about what induces negative thermal expansion. However, we can explain the cause in the same way. Cooling of water may generate denser proper water complexes [41], a denser quartz-like structure [42], hydrogen bond bending [43], or the filling of cavities [44, 45]. Their excess internal energies become higher because of the high values of the soft-repulsive potential near the hard-core contact due to condensation. The coefficient $\alpha$ becomes negative, resulting in negative thermal expansion, which may generate less dense and ice-like complexes [41], less dense tridymitelike ones [42], stretching of the hydrogen bond bending [43], or preventing the cavities from filling and causing negative expansion $[44,45]$.

It is well-known that almost none of the ideas put forward [41-54] explain what causes the negative thermal expansion at temperatures below $4^{\circ} \mathrm{C}$. For example, one claim is that the tetrahedral structure of ice causes the density anomaly, but there is no evidence for this. As a counter analogy, consider a folding umbrella. To open or close it, one pushes or pulls the base of the frame with hand power. The frame has no power to open or close itself without human intervention. In the case of the umbrella, the direct cause of its expansion and contraction is human hand power and not the frame itself. To clarify the thermodynamic mechanism that causes the density anomaly, it is necessary to find what acts as an attractive force to condense water at temperatures above $4^{\circ} \mathrm{C}$, but acts as a repulsive force to expand water below $4^{\circ} \mathrm{C}$ with reducing temperature. Such a force (hereafter referred to for simplicity as the "anomaly force") is the immediate cause of the density anomaly of liquid water. It is difficult to imagine how the tetrahedral structure could create an "anomaly force" analogous to the case of the folding umbrella.

Another suggestion is that hydrogen bonding causes the density anomaly. However, hydrogen bonding is attractive at any temperature and has the tendency to reduce the distance between molecules in thermodynamic equilibrium to condense liquid water. Therefore, it is difficult to consider how hydrogen bonding could turn into a repulsive force below $4^{\circ} \mathrm{C}$ and cause negative thermal expansion.

Regarding the network or clathrate models [10, 20-23], even though it may be plausible that isolated water molecules go into cavities as the temperature lowers to cause liquid water to condense, the models do not explain what makes isolated water molecules leave the filled cavities at temperatures below $4^{\circ} \mathrm{C}$ with reducing temperature and induce negative expansion.

Lactic acid was long believed to be the substance that causes muscle fatigue because it increases with fatigue, but lactic acid was recently found to be a substance that assists in recovery from fatigue. It is now known that active oxygen is the substance responsible for fatigue. Similarly, it cannot be claimed that the density anomaly is caused by some phenomenon just because it accompanies the density anomaly. We can apply this principle to almost every idea put forward until now.

Here, we present the idea that the thermodynamic mechanism found by Yasutomi [40] induces negative thermal expansion. This is accompanied by rearranging orientations of molecules and results in polymorphic structures of solid water depending on the shape of the orientation-dependent potential, or is accompanied by a second critical point, two-state structures, or clathrate structures. In this way, although our study is blind to freezing and, more generally, to the solid phases of the system, it presents significant insights into the thermodynamic properties of water in these phases. We expet that our main remarks may be applicable to a density anomaly in any liquid [39, 40].

We believe that mysteries in thermodynamic phenomena in nature are solved when the intermolecular interactions and the thermodynamic mechanisms that induce the phenomena are elucidated. From this point of view, we show in the present paper that the isothermal compressibility of liquid water is determined by the degree of steepness of the soft-repulsion near the hardcore contact. This helps us to understand the thermodynamic mechanism that causes the compressibility anomaly of liquid water. 


\section{MODELS AND NUMERICAL RESULTS}

We consider a fluid of spherical particles with a pair potential given by a hard-core (HC) repulsion and some tail $\phi(r)$ that is expressed as

$$
\phi(r)= \begin{cases}\infty \quad r<1 & \\ -\sum_{n=2}^{N} a_{n} \frac{\exp \left[-z_{n}(r-1)\right]}{r} & r \geq 1\end{cases}
$$

where $N$ is an arbitrary integer and $z_{n}$ and $a_{n}$ are arbitrary constants. We consider three cases of tails $\phi_{i}(r)(i=1-3)$ and refer to the model with tail $\phi_{i}$ as "Model $\phi_{i}$." The parameters $a_{n}$ are listed in Table 1 . The parameters $z_{n}$ are given by $0.6395(n-1)$, $1.18(n-1)$, and $0.6[1+8(n-2)]$ for $\phi_{1}, \phi_{2}$ and $\phi_{3}$, respectively. Figure 1 shows these potential tails. The diameter $\sigma_{\mathrm{u}}$ of the hard core and the depth $\varepsilon_{\mathrm{u}}$ of the potential are used as units of length and energy, respectively, and are shown in Table 2.

We obtain the thermodynamic properties of the models by using the SCOZA [33-40]. We express the physical quantities by the same symbols, and the numerical computations are performed as described in Yasutomi [39, 40]. Table 2 shows the density grid $\Delta \rho$, the temperature grid $\Delta \beta$, the density $\rho_{0}$ at which we made use of the so-called high-temperature approximation [55] and $\beta_{f}$. Nnumerical computations are performed in the range of $0<\beta<\beta_{f}$.

\section{TABLE 1 | Parameters $a_{n}$ for $\phi_{1}, \phi_{2}$, and $\phi_{3}$.}

\begin{tabular}{cccc}
\hline $\boldsymbol{n}$ & $\boldsymbol{\phi}_{\mathbf{1}}$ & $\boldsymbol{\phi}_{\mathbf{2}}$ & $\boldsymbol{\phi}_{\mathbf{3}}$ \\
$\boldsymbol{a}_{\boldsymbol{n}}$ & $\boldsymbol{a}_{\boldsymbol{n}}$ & $\boldsymbol{a}_{\boldsymbol{n}}$ \\
\hline 2 & +39.5839 & +73.1654 & +1.86973 \\
3 & -433.906 & -320.979 & -53.9562 \\
4 & +1478.56 & -7597.99 & +415.834 \\
5 & -1906.08 & +64001.9 & -1189.08 \\
6 & +791.644 & -134345 & +1456.07 \\
7 & & & -652.705
\end{tabular}

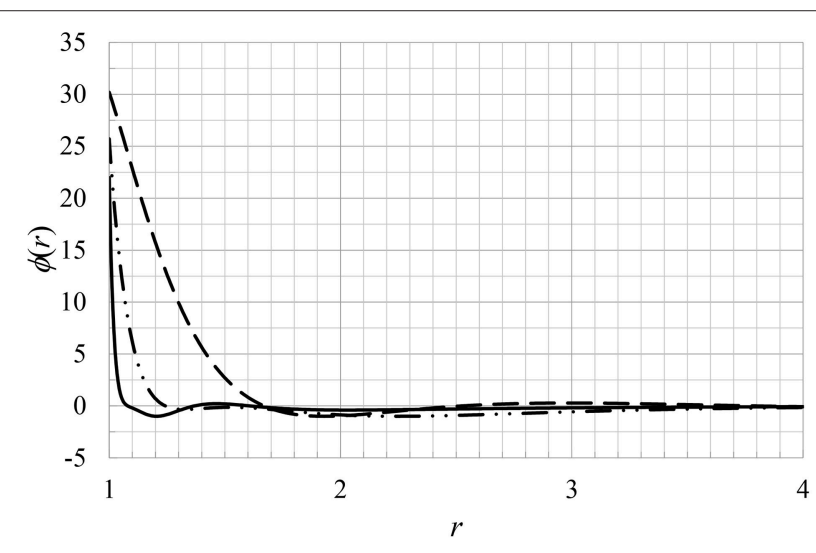

FIGURE 1 | Potential tails. Dashed, chain double-dashed, and solid lines exhibit $\phi_{1}, \phi_{2}$, and $\phi_{3}$, respectively.
All three of the Models $\phi_{1}-\phi_{3}$ reproduce reasonably well the experimentally measured density-temperature relationship of liquid water at 1 bar as shown in Figure 2. The isothermal compressibilities are shown as a function of temperature at 1 bar in Figure 3. The plot demonstrates that the isothermal

TABLE 2 | Parameters used in numerical computations for Models $\phi_{1}-\phi_{3}$.

\begin{tabular}{lccc}
\hline & $\boldsymbol{\phi}_{\mathbf{1}}$ & $\boldsymbol{\phi}_{\mathbf{2}}$ & $\boldsymbol{\phi}_{\mathbf{3}}$ \\
\hline$\Delta \rho$ & 0.001 & 0.001 & 0.0005 \\
$\Delta \beta$ & $2 \times 10^{-10}-10^{-3}$ & $10^{-5}-2 \times 10^{-4}$ & $10^{-7}-10^{-4}$ \\
$\rho_{0}$ & 0.748 & 0.827 & 1.0175 \\
$\beta_{f}$ & 0.310 & 0.244 & 0.332 \\
$\beta_{\mathrm{C}}$ & 0.108 & 0.103 & 0.145 \\
$\rho_{\mathrm{C}}$ & 0.084 & 0.158 & 0.2165 \\
$\varepsilon_{\mathrm{u}}(\mathrm{K})$ & 74.65 & 59.92 & 78.97 \\
$\sigma_{\mathrm{u}}(\AA)$ & 1.965 & 2.156 & 2.402
\end{tabular}

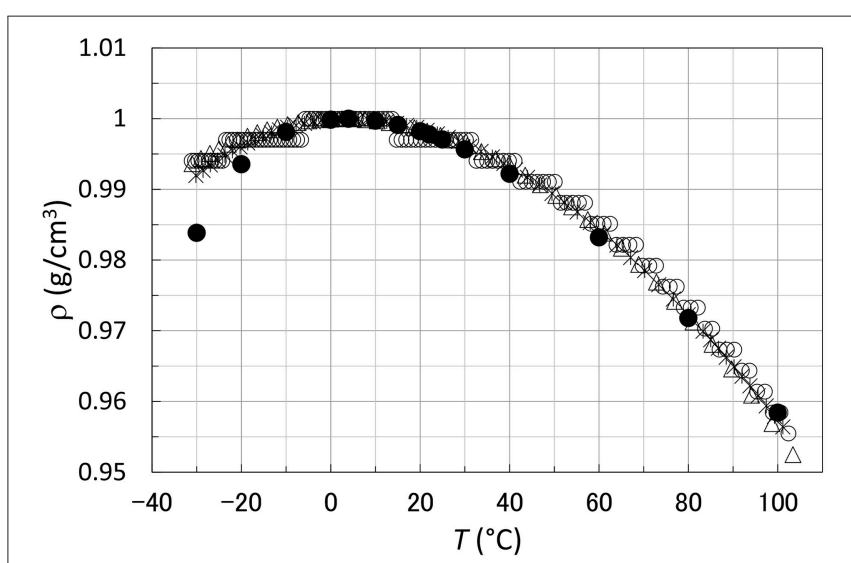

FIGURE 2 | Density-temperature relation of liquid water at $\mathbf{1}$ bar.

Asterisks, triangles, open, and closed circles show those for $\phi_{1}(r), \phi_{2}(r), \phi_{3}(r)$, and experimentally measured data, respectively.

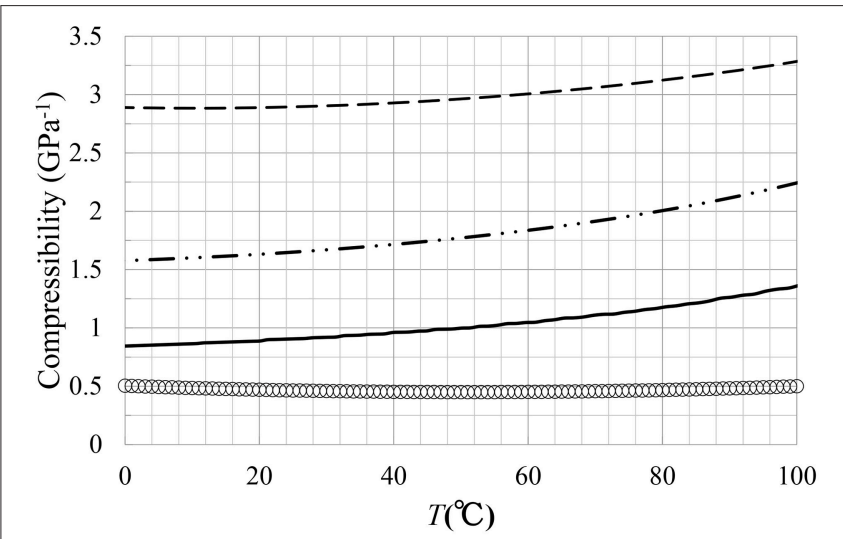

FIGURE 3 | Isothermal compressibility-temperature relation of liquid water at 1 bar. Dashed, chain double-dashed, solid lines, and open circles show those for $\phi_{1}(r), \phi_{2}(r), \phi_{3}(r)$, and experimentally measured data, respectively. 
compressibility reduces with increasing degree of steepness of the potential tail near the hard-core contact.

\section{DISCUSSION}

Thermodynamic quantities can be derived from the excess internal energy $u$ per unit volume defined by

$$
u=2 \pi \rho^{2} \int_{1}^{\infty} d r r^{2} \phi(r) g(r)
$$

where $g(r)$ is a distribution function. The excess internal energy is useful for studying the relationships between the shape of the intermolecular potential and the behavior of a thermodynamic quantity. We have illuminated the thermodynamic mechanism which causes the density anomaly of water through the excess internal energy in our previous paper [40].

The integrand in the above equation shows that the excess internal energy is given by the product of intermolecular interaction $\phi(r)$ and distribution function $g(r)$. This suggests that there are an infinite number of combinations of $\phi(r)$ and $g(r)$ that result in the same $u$, and we have determined many potential tails that reproduce the experimentally measured density anomaly of water at 1 bar in our recent paper [39].

Besides those, we also determined three pair interactions between water molecules (Figure 1), all of which reproduce

\section{REFERENCES}

1. Debenedetti PG. Supercooled and glassy water. J Phys Condens Matter (2003) 15:R1669. doi: 10.1088/0953-8984/15/45/R01

2. Beveridge D, Mezei M, Mehrotra PK, Marchese FT, Ravi-Shankar G, Vasu T, et al. Molecular-based study of fluids. In: Haile JM, Mansoori GA, editors. Advances in Chemistry. Vol. 204. Washington, DC: American Chemical Society (1983). p. 297-351.

3. Jorgensen WL, Chandrasekhar J, Madura JD, Impey RW, Klein ML. Comparison of simple potential functions for simulating liquid water. J Chem Phys. (1983) 79:926-35. doi: 10.1063/1.445869

4. Jorgensen WL, Madura JD. Temperature and Size Dependence for Monte Carlo Simulations of TIP4P Water. Mol Phys. (1985) 56:1381-92. doi: $10.1080 / 00268978500103111$

5. Mahoney MW, Jorgensen WL. A five-site model for liquid water and the reproduction of the density anomaly by rigid, nonpolarizable potential functions. J Chem Phys. (2000) 112:8910-22. doi: 10.1063/1.481505

6. Berendsen HJC, Postma JPM, van Gunsteren WF, Hermans J. Interaction models for water in relation to protein hydration. In: Pullman B, editor. Intermolecular Forces, Volume 14 of The Jerusalem Symposia on Quantum Chemistry and Biochemistry. Netherlands: Springer (1981). p. 331-42. doi: 10.1007/978-94-015-7658-1_21

7. Berendsen HJC, Grigera JR, Straatsma TP. The missing term in effective pair potentials. J Phys Chem. (1987) 91:6269-71. doi: 10.1021/j100308a038

8. Stanley HE, Buldyrev SV, Giovambattista N, Nave EL, Mossa S, Scala A, et al. Application of Statistical Physics to Understand Static and Dynamic Anomallies in Liquid Water. J Stat Phys. (2003) 110:1039-54. doi: 10.1023/A:1022188608924

9. Guillot B. A reappraisal of what we have learnt during three decades of computer simulations on water. J Mol Liquids. (2002) 101:219-60. doi: 10.1016/S0167-7322(02)00094-6

10. Molinero V, Moore EB. Water modeled as an intermediate element between carbon and silicon. J Phys Chem B (2009) 113:4008-16. doi: $10.1021 /$ jp805227c the experimentally measured density anomaly at 1 bar with reasonable accuracy (Figure 2). Figures 2, 3 show that the degree of steepness of the tail near the hard-core contact determines the isothermal compressibility of a liquid. Among the models, Model $\phi_{3}$ best reproduces the experimental data for liquid water. Therefore, tails steeper than $\phi_{3}$ will likely reproduce the experimental data with even better accuracy. It will take much more calculation time to determine the functional representations of such potential tails because the steeper tails should be expressed with a smaller radial distance grid. In addition. numerical computations should be performed with smaller density and temperature grids to attain a more optimal accuracy. Nevertheless, in the comming years we should increasingly be able to discover potential tails that will reproduce the experimental data of both the density-temperature and compressibility-temperature relations at 1 bar with reasonable accuracy. These potential tails will help us to illuminate the physics underlying the numerous anomalies of liquid water. Our main remarks may be applicable to a density anomaly and a thermal compressibility in any liquid [39, 40].

\section{AUTHOR CONTRIBUTIONS}

The author confirms being the sole contributor of this work and approved it for publication.
11. Limmer DT, Chandler D. The putative liquid-liquid transition is a liquid-solid transition in atomistic models of water. J Chem Phys. (2011) 135:134503. doi: $10.1063 / 1.3643333$

12. Abascal JLF, Vega C. A general purpose model for the condensed phases of water: TIP4P/2005. J Chem Phys. (2005) 123:234505. doi: 10.1063/1.2121687

13. Abascal JLF, Vega C. Widom line and the liquid-liquid critical point for the TIP4P/2005 water model. J Chem Phys. (2010) 133:234502. doi: $10.1063 / 1.3506860$

14. Abascal JLF, Vega C. Note: equation of state and compressibility of supercooled water: simulations and experiment. J Chem Phys. (2011) 134:186101. doi: $10.1063 / 1.3585676$

15. Holten V, Bertrand CE, Anisimov MA, Sengers JV. Thermodynamics of supercooled water. J Chem Phys. (2012) 136:094507. doi: 10.1063/1.3690497

16. Overduin SD, Patey GN. An analysis of fluctuations in supercooled TIP4P/2005 water. J Chem Phys. (2013) 138:184502. doi: 10.1063/1. 4803868

17. Helena LP, Aragones JL, Vega C, Noya EG, Abascal JLF, Gonzalez $\mathrm{MA}$, et al. Anomalies in water as obtained from computer simulations of the TIP4P/2005 model: density maxima, and density, isothermal compressibility and heat capacity minima. Mol Phys. (2009) 107:365-74. doi: 10.1080/00268970902784926

18. Vega C, Abascal JLF, Conde MM, Aragones JL. What ice can teach us about water interactions: a critical comparison of the performance of different water models. Faraday Discuss. (2009) 141:251-76. doi: 10.1039/B805531A

19. Russo J, Tanaka H. Understanding water's anomalies with locally favoured structures. Nat Commun. (2014) 5:3556. doi: 10.1038/ncomms4556

20. Oliveira ABD, Netz PA, Barbosa MC. An ubiquitous mechanism for water-like anomalies. EPL (2009) 85:36001. doi: 10.1209/0295-5075/85/36001

21. Gribova NV, Fomin YD, Frenkel D, Ryzhov VN. Water like thermodynamic anomalies in a repulsive-shoulder potential system. Phys Rev E (2009) 79:051202. doi: 10.1103/PhysRevE.79.051202

22. Fomin YD, Tsiok EN, Ryzhov VN. Inversion of sequence of diffusion and density anomalies in core-softened systems. J Chem Phys. (2011) 135:234502. doi: 10.1063/1.3668313 
23. Sadr-Lahijany MR, Scala A, Buldyrev SV, Stanley HE. Liquid-State Anomalies and the Stell-Hemmer Core-Softened Potential. Phys Rev Lett. (1998) 81:4895-8. doi: 10.1103/PhysRevLett.81.4895

24. Wilding NB, Magee JE. Phase behavior and thermodynamic anomalies of core-softened fluids. Phys Rev E (2002) 66:031509. doi: 10.1103/PhysRevE.66.031509

25. Gibson HM, Wilding NB. Metastable liquid-liquid coexistence and density anomalies in a core-softened fluid. Phys Rev E (2006) 73:061507. doi: 10.1103/PhysRevE.73.061507

26. Caballero JB, Puertas AM. Density anomaly and liquid-liquid transition from perturbation theories. Phys Rev E (2006) 74:051506. doi: 10.1103/PhysRevE.74.051506

27. Lomba E, Almarza NG, Martin C, McBridge C. Phase behavior of attractive and repulsive ramp fluids: integral equation and computer simulation studies. J Chem Phys. (2007) 126:244510. doi: 10.1063/1.2748043

28. Jagla EA. Low-temperature behavior of core-softened models: water and silica behavior. Phys Rev E (2001) 63:061509. doi: 10.1103/PhysRevE.63.061509

29. Yan Z, Buldyrev SV, Giovambattista N, Stanley HE. Structural Order for One-Scale and Two-Scale Potentials. PRL (2005) 95:130604. doi: 10.1103/PhysRevLett.95.130604

30. Yan Z, Buldyrev SV, Kumar P, Giovambattista N, Stanley HE. Correspondence between phase diagrams of the TIP5P water model and a spherically symmetric repulsive ramp potential with two characteristic length scales. Phys Rev E (2008) 77:042201. doi: 10.1103/PhysRevE.77. 042201

31. Prestipino S, Saija F, Malescio G. Anomalous phase behavior in a model fluid with only one type of local structure. J Chem Phys. (2010) 133:144504. doi: 10.1063/1.3499830

32. Malescio G, Prestipino S, Saija F. Anomalous melting and solid polymorphism of a modified inverse-power potential. Mol Phys. (2011) 109:2837-44. doi: $10.1080 / 00268976.2011 .609146$

33. Pini D, Stell G, Wilding NB. A liquid-state theory that remains successful in the critical region. Mol Phys. (1998) 95:483-94. doi: $10.1080 / 00268979809483183$

34. Pini D, Stell G, Dickman R. Thermodynamically self-consistent theory of structure for three-dimensional lattice gases. Phys Rev E (1998) 57:2862-71. doi: 10.1103/PhysRevE.57.2862

35. Schöll-Paschinger E. Self-consistent Ornstein-Zernike approximation for the Sogami-Ise fluid. J Chem Phys. (2004) 120:11698-711. doi: 10.1063/1.1755192

36. Schöll-Paschinger E. Vapor-liquid equilibrium and critical behavior of the square-well fluid of variable range: A theoretical study. J Chem Phys. (2005) 123:234513. doi: 10.1063/1.2137713

37. Betancourt-Cárdenas FF, Galicia-Luna LA, Benavides AL, Ramirez JA, SchöllPaschinger E. Thermodynamics of a long-range triangle-well fluid. Mol Phys. (2008) 106:113-26. doi: 10.1080/00268970701832397

38. Yasutomi M. A self-consistent Ornstein-Zernike approximation for a fluid with a screened power series interaction. J Chem Phys. (2010) 133:154115. doi: $10.1063 / 1.3503590$

39. Yasutomi M. Interparticle interactions between water molecules. Front Phys. (2014) 2:64. doi: 10.3389/fphy.2014.00064

40. Yasutomi M. Thermodynamic mechanism of density anomaly of liquid water. Front Phys. (2015) 3:8. doi: 10.3389/fphy.2015.00008
41. Röntgen WC. Ueber die Constitution des flüssigen Wassers. Annalen der Physik. (1892) 281:91-7. doi: 10.1002/andp.18922810108

42. Bernal JD, Fowler RH. A theory of water and ionic solution, with particular reference to hydrogen and hydroxyl ions. J Chem Phys. (1933) 1:515-48. doi: $10.1063 / 1.1749327$

43. Pople JA. Molecular association in liquids. II. a theory of the structure of water. Proc R Soc Lond A (1951) 205:163-78. doi: 10.1098/rspa.1951.0024

44. Samoilov OY. A new approach to the study of hydrogen of ions in aqueous solutions. Discuss Faraday Soc. (1957) 24:141-6. doi: 10.1039/df9572400141

45. Pauling L. The structure of water. In: Hadzi D, Thompson HW, editors. Hydrogen Bonding. London: Pergamon Press Ltd (1959). p. 1-6.

46. Bernal JD. The Bakerian lecture, 1962. The structure of liquids. Proc R Soc Lond A. (1964) 280:299-322. doi: 10.1098/rspa.1964.0147

47. Sceats MG, Rice SA. The water-water pair potential near the hydrogen bonded equilibrium configuration. J Chem Phys. (1980) 72:3236-47. doi: $10.1063 / 1.439560$

48. Sceats MG, Rice SA. A random network model calculation of the free energy of liquid water. J Chem Phys. (1980) 72:6183-91. doi: 10.1063/1.439077

49. Rice SA, Sceats MG. A random network model for water. J Chem Phys. (1981) 85:1108-19. doi: 10.1021/j150609a009

50. Némety G, Scheraga HA. Structure of water and hydrophobic bonding in proteins. I. A model for the thermodynamic properties of liquid water. J Chem Phys. (1962) 36:3382-400. doi: 10.1063/1.1732472

51. Stanley HE. A polychromatic correlated-site percolation problem with possible relevance to the unusual behaviour of supercooled $\mathrm{H} 2 \mathrm{O}$ and $\mathrm{D} 2 \mathrm{O}$. J Phys A Math Gen. (1979) 12:L329. doi: 10.1088/0305-4470/12/12/003

52. Malenkov G. Liquid water and ices: understanding the structure and physical properties. J Phys Condens Matter. (2009) 21:283101. doi: 10.1088/0953$8984 / 21 / 28 / 283101$

53. Poole PH, Sciortino R, Essmann U, Stanley HE. Phase behaviour of metastable water. Nature (1992) 360:324-28. doi: 10.1038/360324a0

54. Chatterjee S, Debenedetti PG, Stillinger FH, Lynden-Bell RM. A computational investigation of thermodynamics, structure, dynamics and solvation behavior in modified water models. J Chem Phys. (2008) 128:124511. doi: 10.1063/1.2841127

55. Hansen JP, McDonald IR. Theory of Simple Liquids. New York, NY: Academic Press (1986).

Conflict of Interest Statement: The author declares that the research was conducted in the absence of any commercial or financial relationships that could be construed as a potential conflict of interest.

The reviewer JG and handling Editor declared their shared affiliation, and the handling Editor states that the process nevertheless met the standards of a fair and objective review.

Copyright (c) 2016 Yasutomi. This is an open-access article distributed under the terms of the Creative Commons Attribution License (CC BY). The use, distribution or reproduction in other forums is permitted, provided the original author(s) or licensor are credited and that the original publication in this journal is cited, in accordance with accepted academic practice. No use, distribution or reproduction is permitted which does not comply with these terms. 